\title{
Melagatran for thromboprophylaxis after mechanical valve implantation: Results in a heterotopic porcine model
}

Jess L. Thompson, MD, ${ }^{a}$ Chad E. Hamner, MD, ${ }^{a}$ D. Dean Potter, MD, ${ }^{a}$ Matthew Lewin, MD, ${ }^{b}$ Thoralf M. Sundt, MD, and Hartzell V. Schaff, MD

From the Division of Cardiovascular Surgery $^{\mathrm{a}}$ and the Department of Laboratory Medicine and Pathology, ${ }^{\mathrm{b}}$ Mayo Clinic, Rochester, Minn.

AstraZeneca International provided melagatran and St Jude Medical, Inc, provided valved conduits. Both companies provided grant support for this study. The Mayo Clinic Division of Cardiovascular Surgery holds Technology Licensing Agreements with St Jude Medical, Inc.

Received for publication Oct 24, 2006; revisions received Jan 2, 2007; accepted for publication Jan 17, 2007.

Address for reprints: Hartzell V. Schaff, MD, Division of Cardiovascular Surgery, Mayo Clinic, 200 First St SW, Rochester, MN 55905 (E-mail: schaff.hartzell@ mayo.edu).

J Thorac Cardiovasc Surg 2007;134:359-65

$0022-5223 / \$ 32.00$

Copyright $(9) 2007$ by The American Association for Thoracic Surgery

doi:10.1016/j.jtcvs.2007.01.093
Objective: Melagatran, the active form of ximelagatran, is a novel, direct thrombin inhibitor that does not have a narrow therapeutic window regarding hemorrhagic and thromboembolic events. We aimed to determine whether melagatran would be effective in preventing thrombus formation on heterotopically placed mechanical heart valves.

Methods: A graft containing a bileaflet mechanical heart valve was implanted in the descending thoracic aorta of domestic swine. Two groups of 6 animals received daily subcutaneous injections of either melagatran $(2.4 \mathrm{mg} / \mathrm{kg}, 3$ times per day) or dalteparin ( $175 \mathrm{U} / \mathrm{kg}, 2$ times per day) for 30 days. Four control animals received no anticoagulation therapy. Fecal HemoQuant and serum hemoglobin levels were recorded. Thirty days after the procedure, platelets were labeled with indium 111, the abdominal organs were inspected, and thrombi and platelets deposited on the valve were measured.

Results: Median thrombus burden on the valves was $0.4 \mathrm{mg}$ (interquartile range, 0.15-5.45 mg) with melagatran, $0.5 \mathrm{mg}$ (interquartile range, $0-14.5 \mathrm{mg}$ ) with dalteparin, and $168 \mathrm{mg}$ (interquartile range, $32.5-665.75 \mathrm{mg}$ ) for controls (melagatran vs dalteparin and control; $P=.04$ ). Median platelet deposition on the valves was 0 (interquartile range, $0-8.9 \times 10^{4}$ ) with melagatran, $49.9 \times 10^{4}$ (interquartile range, $27.9 \times 104-191.8 \times 10^{4}$ ) with dalteparin, and $115.2 \times 10^{4}$ (interquartile range, $9.6 \times 10^{4}-243 \times 10^{4}$ ) for controls (melagatran vs dalteparin and control; $P=.02$ ). Melagatran did not increase the risk of thromboembolism or bleeding.

Conclusions: Thrombus and platelet accumulation on the prosthetic valves was decreased by melagatran and dalteparin. The use of melagatran or other related direct thrombin inhibitors warrants further study in prophylaxis of thromboembolism in patients with mechanical heart valves.

$\mathrm{F}$ or more than 45 years, vitamin $\mathrm{K}$ antagonists such as warfarin have been used to prevent thromboembolic complications in patients with mechanical heart valve prostheses. Low-molecular-weight heparins (LMWH) have also been used for thromboembolism prophylaxis, generally for short-term therapy. The oral and subcutaneous anticoagulants used at present have several limitations that make their use in the outpatient setting cumbersome. For example, routine invasive monitoring is required to maintain therapeutic levels of warfarin owing to its narrow therapeutic range. Further, warfarin has a slow onset of action and has the potential for numerous interactions with other medications and foods. Invasive monitoring is also required for unfractionated heparin, and an injection is needed for prophylaxis with LMWH.

Ximelagatran, the oral prodrug of melagatran, is the first orally administered direct thrombin inhibitor to reach phase III clinical trials. It has several advantages over the currently approved anticoagulants, including its availability in both paren- 


\author{
Abbreviations and Acronyms \\ aPTT = activated partial thromboplastin time \\ $\mathrm{Hb}=$ hemoglobin \\ IQR = interquartile range \\ LMWH $=$ low-molecular-weight heparin
}

teral and oral formulations, prompt onset of action, predictable dose response (no coagulation monitoring necessary), virtually no interaction with food, and a low potential for interaction with other medications. ${ }^{1}$ Ximelagatran has been evaluated for several potential indications, including for the treatment $^{2}$ and prevention ${ }^{3,4}$ of venous thromboembolism, for the prevention of cardioembolic events in patients with nonvalvular atrial fibrillation, ${ }^{5}$ and for decreasing mortality in patients who have had a recent myocardial infarction. ${ }^{6}$ However, no data exist in the literature regarding the effectiveness of either ximelagatran or melagatran in preventing thrombotic complications from mechanical heart valve prostheses. We aimed to determine whether melagatran is effective in preventing thrombus formation on heterotopically placed mechanical heart valves.

\section{Materials and Methods}

\section{Anesthesia and Surgical Preparation}

Heterotopic, valved conduit implantation was performed in 16 domestic swine (weight, 35-45 kg). Each animal was randomly assigned to receive 1 of 3 anticoagulation options: 6 received melagatran (AstraZeneca R\&D, Mölndal, Sweden), 6 received dalteparin sodium (Pfizer, New York, NY), and 4 animals received no anticoagulation therapy. The study protocol was approved by the Mayo Foundation Institutional Animal Care and Use Committee, and the animals received humane care in compliance with the "Guide for the Care and Use of Laboratory Animals" (NIH Publication No. 85-23).

\section{Operative Procedure}

A 9.6F single-lumen cuffed Hickman catheter (Bard Access Systems, Salt Lake City, Utah) was placed into the left external jugular vein and tunneled subcutaneously to exit the skin between the scapulas. Proper location was confirmed fluoroscopically.

The valved conduit was constructed from a 19-mm SJM Masters Series aortic valved graft (St Jude Medical, Inc, Minneapolis, Minn). A left thoracotomy in the fifth intercostal space was performed, and the descending aorta was exposed. The animal was heparinized $(300 \mathrm{U} / \mathrm{kg})$, and a side-biting clamp was placed onto the distal thoracic aorta (Figure 1). Maintenance of a blood pressure reading from the femoral arterial line ensured distal flow through the aorta. The aorta was incised and the efferent limb of the graft anastomosed to the distal thoracic aorta with a running 4-0 polypropylene suture. This procedure was repeated for the proximal aorta and afferent limb of the graft. The graft was deaired by filling it with blood before the final suture was tied in place. The bypassed portion of the native aorta was then ligated with 2 umbilical tapes. Anticoagulation was reversed with protamine (0.5-1 mg/100 U heparin), and a 28F chest tube was inserted. The

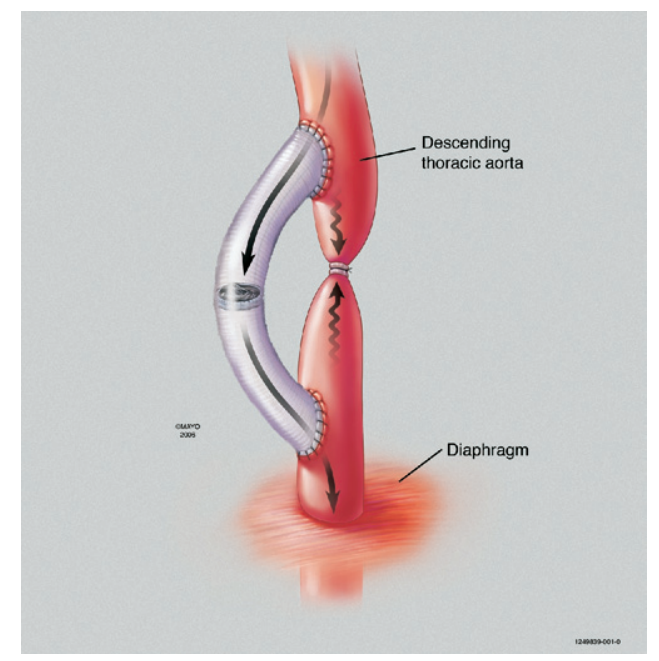

Figure 1. Cartoon depicting the heterotopic model. A valved graft has been implanted onto the aorta, with diversion of the bloodstream through the conduit by ligation of the aorta. (Used with permission of Mayo Foundation for Medical Education and Research.)

chest was then closed in layers. After the animal was extubated, fluoroscopy of the valve was obtained to ascertain leaflet motion.

\section{Anticoagulation Protocol}

Target levels of anticoagulation were set in accordance with human standards: specifically, a prolongation of the activated partial thromboplastin time (aPTT) to 1.5 to 2 times baseline for the direct thrombin inhibitor (melagatran) group ${ }^{7}$ and an elevation of the anti-Xa activity level (with the factor Xa inhibition test) to 0.6 to $1.0 \mathrm{IU} / \mathrm{mL}$ for the LMWH (dalteparin) group. ${ }^{8}$ Anticoagulant administration was begun the morning of the first postoperative day and continued for 30 days. The melagatran group $(n=6)$ received $2.4 \mathrm{mg} / \mathrm{kg}$ melagatran subcutaneously every 8 hours; the LMWH group $(n=6)$ received dalteparin at $175 \mathrm{U} / \mathrm{kg}$ subcutaneously every 12 hours. The control group $(n=4)$ did not receive anticoagulation.

\section{Long-Term Care}

All animals were followed up at our in-house animal care facility for the 30-day duration of the study, where they were monitored daily by the investigators and a veterinary technician. The animals were weighed every 3 days to maintain the appropriate weightbased dose of anticoagulation therapy. Stool HemoQuant and serum hemoglobin $(\mathrm{Hb})$ levels were determined weekly. A HemoQuant level less than $2 \mathrm{mg} \mathrm{Hb}$ per gram stool was considered normal $^{9}$ and was also compared with the HemoQuant levels of 12 pigs that had not received any anticoagulation or operative intervention.

The aPTT and anti-Xa activity levels were determined by serial blood draws performed on postoperative days 4, 15, and 29. The blood was sampled immediately before anticoagulant administration and then $0.5,1,2$, and 4 hours after administration. All 
laboratory studies were performed in the standard manner of the Mayo Medical Laboratories (Mayo Clinic, Rochester, Minn).

So that platelet deposition on the valves could be quantified, animals underwent autologous platelet labeling with indium $111 .^{10}$ After the labeling, the animals were anesthetized, placed on mechanical ventilation, and treated with heparin. A complete blood count was obtained. The mechanical valve was explanted and the conduit was removed from the valve. The valve was placed into a scintillation counter, and the level of radioactivity was recorded. Background level of radioactivity was also recorded, as was the amount of radioactivity emanating from 2 10-mL tubes of blood obtained at the time the animals were put to death. The average amount of radioactivity from one platelet could be determined with the knowledge of the platelet count (platelet radioactivity $=$ blood radioactivity/[blood volume $\times$ platelet count]). The number of platelets deposited $=$ valve radioactivity/platelet radioactivity. Finally, any thrombus on the valve was removed and weighed.

A laparotomy was performed, and the liver, spleen, and large and small bowel were inspected grossly for evidence of thromboembolic or bleeding events. Both kidneys were inspected grossly, explanted, and then examined microscopically for evidence of thromboembolism.

\section{Statistical Analysis}

All analyses were conducted with SAS version 8.2 (SAS Institute, Inc, Cary, NC). Continuous data are expressed as mean \pm SEM. Means of 2 groups were compared with the rank sum test. Means of multiple groups were compared with the Kruskal-Wallis test.

\section{Results}

The coagulation profiles showed that clinically relevant levels of anticoagulation were achieved in both experimental groups. On the fourth postoperative day, the mean baseline aPTT of the animals that received melagatran was $79 \pm$ 34 seconds and doubled to $159 \pm 29$ seconds 15 minutes after administration. The aPTT remained elevated through 30 minutes at $139 \pm 28$ seconds and decreased to $45 \pm 11$ seconds by 2 hours. The mean baseline anti-Xa activity level on the fourth postoperative day for the animals that received dalteparin was $0.08 \pm 0.01 \mathrm{IU} / \mathrm{mL}$ and rose to $0.63 \pm 0.12 \mathrm{IU} / \mathrm{mL}$ at 1 hour. The elevation was prolonged past 4 hours $(0.36 \pm 0.04 \mathrm{IU} / \mathrm{mL})$. More pronounced anticoagulation response was obtained the longer the animals received anticoagulation.

Differences in thrombus deposition were evident on gross inspection of the explanted valves (Figure 2). This difference was confirmed by measuring the weight of the thrombus and the number of platelets deposited onto the valves. Median thrombus burden on the valves was $0.4 \mathrm{mg}$ (interquartile range [IQR], $0.15-5.45 \mathrm{mg}$ ) for the melagatran group, $0.5 \mathrm{mg}$ (IQR, 0-14.5 mg) for the dalteparin group, and $168 \mathrm{mg}$ (IQR, 32.5-665.75 mg) for the control group (Figure 3). The overall difference in the thrombus weights among the 3 groups did not reach significance $(P=.08)$. A statistically significant difference was found when the mel- agatran group was compared with the dalteparin and control groups combined $(P=.04)$.

The median number of platelets deposited on the valves was $0\left(\mathrm{IQR}, 0-8.9 \times 10^{4}\right)$ for the melagatran group, $49.9 \times$ $10^{4}$ (IQR, $27.9 \times 10^{4}-191.8 \times 10^{4}$ ) for the dalteparin group, and $115.2 \times 10^{4}\left(\mathrm{IQR}, 9.6 \times 10^{4}-243 \times 10^{4}\right)$ for the controls (melagatran group vs dalteparin or control group; $P=.02)$.

Melagatran did not increase the risk of bleeding events compared with dalteparin. The mean fecal $\mathrm{Hb}$ level was $0.58 \pm 0.23 \mathrm{mg} \mathrm{Hb}$ per gram stool for the melagatran group, $0.57 \pm 0.12 \mathrm{mg} \mathrm{Hb}$ per gram stool for the dalteparin group, and $0.62 \pm 0.17 \mathrm{mg} \mathrm{Hb}$ per gram stool for the control group $(P=.70)$. On postoperative day 1 , the mean serum $\mathrm{Hb}$ of the dalteparin group was $9 \pm 0.9 \mathrm{~g} / \mathrm{dL}$, which was lower than that of the melagatran group (10.8 \pm $0.3 \mathrm{~g} / \mathrm{dL}$ ). By day 29 of the study, the serum Hb levels were almost equal: melagatran group $11.8 \pm 0.5 \mathrm{~g} / \mathrm{dL}$ and dalteparin group $12.2 \pm 0.6 \mathrm{~g} / \mathrm{dL}(P=.70)$.

At necropsy, neither gross inspection of the abdominal organs nor microscopic examination of the kidneys demonstrated evidence of thromboembolism in any of the groups.

\section{Discussion}

To our knowledge, this is the first report of a direct thrombin inhibitor being used for thromboprophylaxis for mechanical heart valves. We hypothesized that melagatran could be used for thromboembolism prophylaxis for mechanical heart valve prostheses. Data exist supporting the long-term use of LMWH in patients with mechanical heart valves. ${ }^{11}$ We chose not to use warfarin because previous attempts to use it in a porcine model have not been successful. Specifically, Grehan and associatees ${ }^{12}$ observed a prohibitively high number of early deaths when using warfarin in pigs.

The pharmacokinetic properties of melagatran have been shown to be stable and reproducible, which supports fixeddose administration without routine coagulation monitoring. Specific circumstances (eg, emergencies, research) may arise, however, in which the clinician may want to determine the anticoagulation effect of melagatran and will need to know which coagulation assay to order. By inhibiting the thrombin-mediated conversion of fibrinogen to fibrin (one of the last steps of the final common pathway of the coagulation cascade), melagatran affects all of the coagulation assays. ${ }^{1}$ Although the aPTT is relatively insensitive to melagatran concentration, it does provide a qualitative indication of the anticoagulation effect. Furthermore, prolongation of the aPTT is consistent over a wide range of patient demographics and disease states and when taken with other drugs and food.

The thrombin time assay is very sensitive to melagatran concentrations, but defining a "normal" thrombin time may be difficult and requires the establishment of standard 

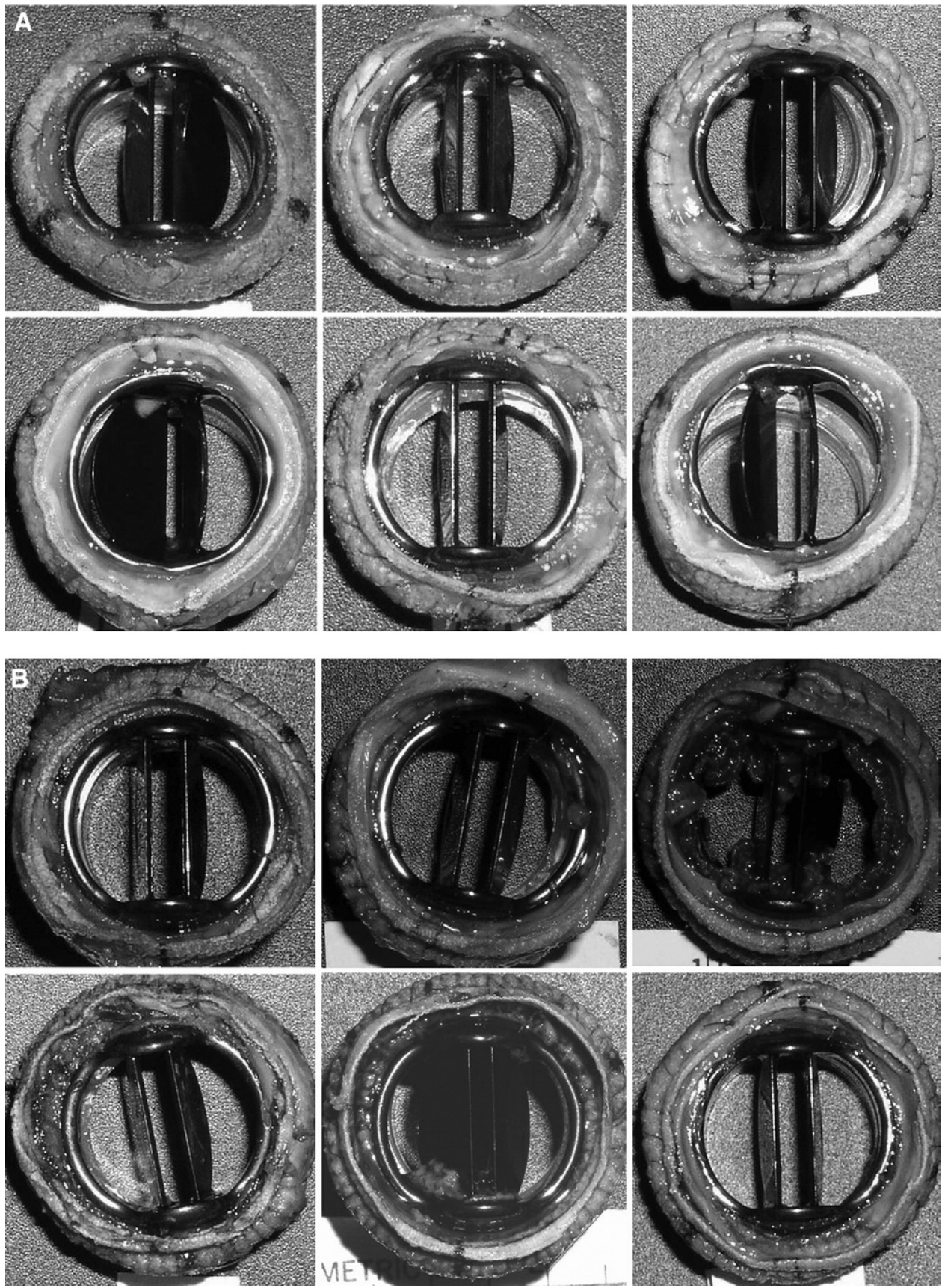

Figure 2. Photographs illustrating thrombus accumulation on the explanted valves $\mathbf{3 0}$ days after implantation in animals receiving melagatran (A), dalteparin (B), or no anticoagulation therapy (C). Visual inspection shows that both melagatran and dalteparin decrease thrombus formation.

curves locally. In general, there is limited experience with using the activated clotting time in clinical trials, and the prothrombin time/international normalized ratio gives unpredictable results. Although other tests (ecarin clotting time, prothrombinase-induced clotting time) may be of value in determining the extent of anticoagulant effect, they do not have the wide clinical availability of either the aPTT or the thrombin time assays.

In the present study, thrombus accumulation on the prosthetic valves was reduced by melagatran and dalteparin, which was confirmed visually and by thrombus weight. Fewer platelets were deposited on the valves of the melagatran group, 

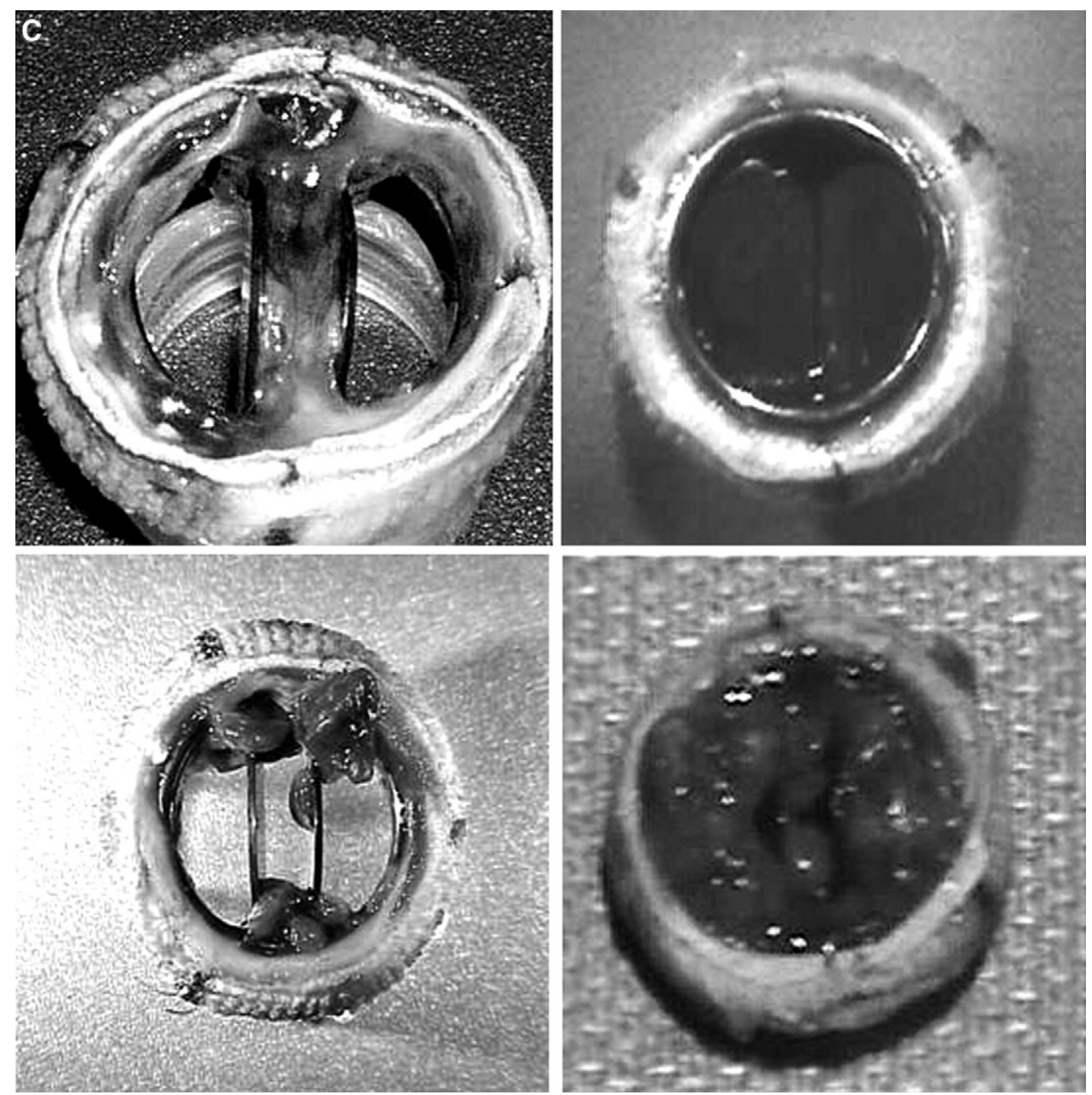

Figure 2. Continued

which may be explained by the platelet-inhibiting action of this direct thrombin inhibitor. Melagatran did not increase risk of distal thromboembolism or bleeding compared with dalteparin, which mirrors the results of recent large clinical trials. ${ }^{13,14}$

The porcine model is particularly well suited for studying novel anticoagulants. The fibrinolytic mechanisms ${ }^{15}$ and platelet function $^{16}$ of humans are more similar to swine than they are to sheep, and swine have been used in preclinical studies evaluating the thrombogenicity of coronary artery stents and vascular prostheses. ${ }^{17,18}$ Although the sheep model has been used for determining the performance characteristics of valvular prostheses, ${ }^{19}$ it did not predict the thrombotic potential of the Medtronic Parallel valve (Medtronic, Inc, Minneapolis, Minn). ${ }^{20}$ For these reasons, the thromboembolism prophylaxis capacity of novel anticoagulants probably should not be tested in the ovine model.

We believe that a heterotopically placed mechanical valve is an appropriate model for screening the thrombo- embolic prophylaxis potential of novel anticoagulants. The use of a heterotopic model eliminates many of the technical complications and expenses associated with cardiopulmonary bypass. Also, the substantial somatic growth of pigs (1-2 kg/d) raises the concern of perivalvular leaks and prosthesis-to-animal size mismatch, which is obviated by a heterotopic model. The use of Yucatan minipigs has been advocated as a means to avoid the somatic growth issue ${ }^{21}$ but has not been extensively used because of the high cost of this strain.

The heterotopic model we used subjects the valve to the continuous forward stream of blood in the descending aorta, causing the leaflets to remain in the open position. Postoperative fluoroscopy demonstrated that the valve leaflets remained continuously in the open position. It has been hypothesized that high-leak jet velocities at the time of valve closure create a "door-slamming" effect that may keep the hinge regions free of deposited blood elements. ${ }^{22}$ Lacking 


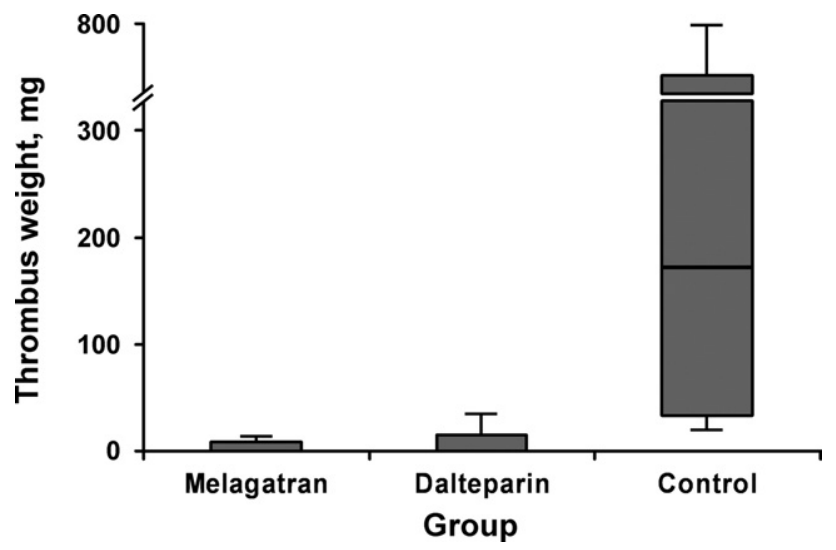

Figure 3. Box plots showing median (interquartile range) thrombus weight for the melagatran, dalteparin, and control groups. (Melagatran vs dalteparin and control, $P=.04$.)

the thrombus-clearing mechanisms of moving leaflets, a mechanical valve heterotopically placed may be at increased risk for thrombus formation compared with an orthotopically placed valve.

The US Food and Drug Administration has not approved ximelagatran for use in humans. ${ }^{23}$ Transient elevated levels of alanine aminotransferase during long-term administration remain the principal source of concern regarding its usage. ${ }^{24}$ Ximelagatran (Exanta; AstraZeneca) was approved by the European regulatory authorities in May 2004 for short-term use in patients undergoing hip- or knee-replacement surgery, ${ }^{25}$ but it has subsequently been voluntarily withdrawn by its manufacturer for ongoing concerns of liver toxicity. ${ }^{26}$ Although ximelagatran is the first of its class to reach phase III clinical trials, novel direct thrombin inhibitors are already being developed. ${ }^{27}$

This study was limited in duration and in the number of animals. Future studies should address these limitations, in addition to developing a model for orthotopic valve implantation and searching for a porcine model of stable anticoagulation with warfarin.

In conclusion, as novel anticoagulants are being developed, a model in which to evaluate their thromboembolic prophylaxis efficacy must be developed. We used a heterotopically placed valved conduit in a porcine model to evaluate the direct thrombin inhibitor melagatran, which we compared with dalteparin and no anticoagulation. We demonstrated that melagatran is equivalent to dalteparin in preventing thrombus formation on mechanical heart valves and that it does not increase the risk for thromboembolic or bleeding events. This study showed that a heterotopic model of mechanical heart valve implantation is technically achievable and sufficiently demanding to screen the thromboembolism prophylaxis efficacy of novel anticoagulants.
In the future, direct thrombin inhibitors may have an important role in thromboprophylaxis for patients with mechanical valves.

\section{References}

1. Carlsson SC, Mattsson C, Eriksson UG, Sarich TC, Wahlander K, Eliasson $\mathrm{A}$, et al. A review of the effects of the oral direct thrombin inhibitor ximelagatran on coagulation assays. Thromb Res. 2005;115:9-18.

2. Schulman $\mathrm{S}$. The role of ximelagatran in the treatment of venous thromboembolism. Pathophysiol Haemost Thromb. 2005;34(Suppl 1):18-24.

3. Schulman S, Lundstrom T, Wahlander K, Billing Clason S, Eriksson H. Ximelagatran for the secondary prevention of venous thromboembolism: a complementary follow-up analysis of the THRIVE III study. Thromb Haemost. 2005;94:820-4.

4. Schulman S, Wahlander K, Lundstrom T, Clason SB, Eriksson H Secondary prevention of venous thromboembolism with the oral direct thrombin inhibitor ximelagatran. $N$ Engl J Med. 2003;349:1713-21.

5. Olsson SB, Halperin JL. Prevention of stroke in patients with atrial fibrillation. Semin Vasc Med. 2005;5:285-92.

6. Wallentin L, Wilcox RG, Weaver WD, Emanuelsson H, Goodvin A, Nystrom P, et al. Oral ximelagatran for secondary prophylaxis after myocardial infarction: the ESTEEM randomised controlled trial. Lancet. 2003;362:789-97.

7. Warkentin TE, Greinacher A. Heparin-induced thrombocytopenia: recognition, treatment, and prevention: the Seventh ACCP Conference on Antithrombotic and Thrombolytic Therapy. Chest. 2004; 126:311S-37S.

8. Hirsh J, Raschke R. Heparin and low-molecular-weight heparin: the Seventh ACCP Conference on Antithrombotic and Thrombolytic Therapy. Chest. 2004;126:188S-203S.

9. Ahlquist DA, McGill DB, Schwartz S, Taylor WF, Owen RA. Fecal blood levels in health and disease: a study using HemoQuant. $N$ Engl J Med. 1985;312:1422-8.

10. Dewanjee MJ, Rao SA, Didisheim P. Indium-111 tropolone, a new high-affinity platelet label: preparation and evaluation of labeling parameters. J Nucl Med. 1981;22:981-7.

11. Shapira Y, Sagie A, Battler A. Low-molecular-weight heparin for the treatment of patients with mechanical heart valves. Clin Cardiol. 2002:25:323-7.

12. Grehan JF, Hilbert SL, Ferrans VJ, Droel JS, Salerno CT, Bianco RW Development and evaluation of a swine model to assess the preclinical safety of mechanical heart valves. J Heart Valve Dis. 2000;9:710-9.

13. Fiessinger JN, Huisman MV, Davidson BL, Bounameaux H, Francis $\mathrm{CW}$, Eriksson H, et al. Ximelagatran vs low-molecular-weight heparin and warfarin for the treatment of deep vein thrombosis: a randomized trial. JAMA. 2005;293:681-9.

14. Albers GW, Diener HC, Frison L, Grind M, Nevinson M, Partridge S, et al. Ximelagatran vs warfarin for stroke prevention in patients with nonvalvular atrial fibrillation: a randomized trial. JAMA. 2005;293:690-8.

15. Gross DR. Thromboembolic phenomena and the use of the pig as an appropriate animal model for research on cardiovascular devices. Int J Artif Organs. 1997;20:195-203.

16. Goodman SL. Sheep, pig, and human platelet-material interactions with model cardiovascular biomaterials. J Biomed Mater Res. 1999; 45:240-50.

17. Scott NA, Robinson KA, Nunes GL, Thomas CN, Viel K, King SB III, et al. Comparison of the thrombogenicity of stainless steel and tantalum coronary stents. Am Heart J. 1995;129:866-72.

18. Hazekamp MG, Goffin YA, Huysmans HA. The value of the stentless biovalve prosthesis: an experimental study. Eur J Cardiothorac Surg. 1993;7:514-9.

19. Gallegos RP, Nockel PJ, Rivard AL, Bianco RW. The current state of in-vivo pre-clinical animal models for heart valve evaluation. J Heart Valve Dis. 2005;14:423-32.

20. Bodnar E. The Medtronic Parallel valve and the lessons learned. J Heart Valve Dis. 1996;5:572-3.

21. Smerup M, Pedersen TF, Nyboe C, Funder JA, Christensen TD, Nielsen SL, et al. A long-term porcine model for evaluation of prosthetic heart valves. Heart Surg Forum. 2004;7:E259-64. 
22. Ellis JT, Yoganathan AP. A comparison of the hinge and near-hinge flow fields of the St Jude Medical Hemodynamic Plus and Regent bileaflet mechanical heart valves. J Thorac Cardiovasc Surg. 2000;119:83-93.

23. AstraZeneca International [homepage on the Internet]. AstraZeneca receives action letter from FDA for Exanta ${ }^{\circledR}$ (ximelagatran) [updated 2004 Oct 11; cited 2006 Mar 15]. Available from: http:// www.astrazeneca.com/pressrelease/3285.aspx.

24. Gurewich V. Ximelagatran: promises and concerns. JAMA. 2005;293:736-9.

25. AstraZeneca International [homepage on the Internet]. Successful outcome of the mutual recognition procedure for Exanta ${ }^{\mathrm{TM}}$ (ximelagatran) in
Europe [updated 2004 May 4; cited 2006 Mar 15]. Available from: http://www.astrazeneca.com/pressrelease/1898.aspx.

26. AstraZeneca International [homepage on the Internet]. AstraZeneca decides to withdraw Exanta ${ }^{\mathrm{TM}}$ [updated $2006 \mathrm{Feb}$ 14; cited 2006 Mar 15]. Available from: http://www.astrazeneca.com/pressrelease/5217.aspx.

27. Eriksson BI, Dahl OE, Buller HR, Hettiarachchi R, Rosencher N, Bravo ML, et al. A new oral direct thrombin inhibitor, dabigatran etexilate, compared with enoxaparin for prevention of thromboembolic events following total hip or knee replacement: the BISTRO II randomized trial. J Thromb Haemost. 2005;3:103-11.

Access to The Journal of Thoracic and Cardiovascular Surgery Online is reserved for print subscribers!

Full-text access to The Journal of Thoracic and Cardiovascular Surgery Online is available for all print subscribers. To activate your individual online subscription, please visit The Journal of Thoracic and Cardiovascular Surgery Online, point your browser to http://www.mosby.com/jtcvs, follow the prompts to activate your online access, and follow the instructions. To activate your account, you will need your subscriber account number, which you can find on your mailing label (note: the number of digits in your subscriber account number varies from 6 to 10). See the example below in which the subscriber account number has been circled:

\section{Sample mailing label}

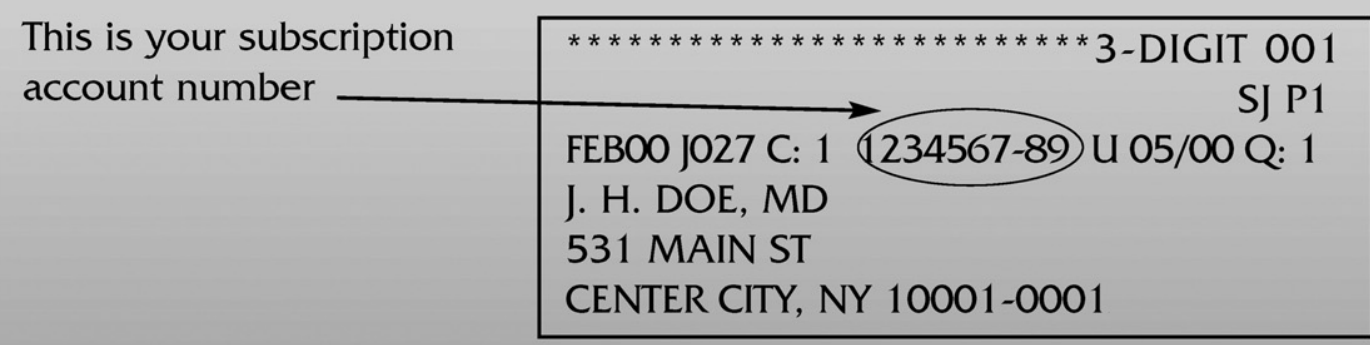

Personal subscriptions to The Journal of Thoracic and Cardiovascular Surgery Online are for individual use only and may not be transferred. Use of The Journal of Thoracic and Cardiovascular Surgery Online is subject to agreement to the terms and conditions as indicated online. 